\title{
On the Delivery Likelihood of Two-Hop Relay MANETs using Erasure Cryptography
}

\author{
Prema Somani \\ M B E Society's College of \\ Engineering, Ambajogai \\ Maharastra, India
}

\author{
B.M. Patil \\ M B E Society's College of \\ Engineering, Ambajogai \\ Maharastra, India
}

\begin{abstract}
In this proposed method a new routing protocol is SelfAdaptive on demand Geographic routing protocols. One of the distinctive features has provide reliable and energy efficient feature to existing schemes in order to increase the lifetime of the network and reduces control overhead compute up to the proactive schemes which provides reliable routing at the same time with a lesser amount of energy utilization. We are embedding optimization (Marko chain algorithm) on the delivery probability performance in a two-hop relay algorithm with erasure coding. The erasure coding used for the message that is erasure coded into multiple frames at source node. Also we developed finite-state Markov chain model to characterize the complicated message delivery process in the MANETs. Based on the above model, closed-form expressions are further derived for the corresponding message delivery probability under any given message lifetime and message size. The above concept is verified through simulation studies, which can be used to accurately predict the message delivery probability behavior and characterize its relationship with the message size.
\end{abstract}

\section{Keywords}

Delivery probability, Two-hop relay, Erasure coding.

\section{INTRODUCTION}

Mobile ad hoc network (MANET) is a peer-to-peer network without any fixed topology or centralized administration, and also fully self-organized mobile node. It is deployed and reconfigured according to its need. It has been observer that it is used in various applications such as the disaster relief, emergency response, daily information exchange, etc. Hence it is crucial component in new generation networks [1], [2]. Similar kind of work has been done in order to understand and address the various deliveries and delay performance issues using different protocols in ad-hoc network. Zhang et al. have been proposed ODE (ordinary differential equations) based framework to analyze delay in the delivery of epidemic routing and its variants. Later Hanbaliet al. study on a multi copy two-hop relay algorithm and evaluate the performance. Later in [5] [6] multi copy two hop relay algorithm are explored the impact of packet lifetime on the packet delivery delay. Recently a new method was introduced in [7] derived closed form expressions for the packet delivery delay of erasure coding and it is enhanced two-hop relay and that of group-based two-hop relay in [8].

The delivery delay work [3]-[8], to deliver a message (or packet) from the source to the destination, having expected time it takes a routing protocol i.e., the unit of time needed to attain the delivery likelihood one, that is truly an easy extreme case of the delivery likelihood study. The corresponding delivery probability under any given message lifetime (permitted delivery delay) is focused on network designs. Further observed that in challenging MANET environment multiple message replicas are usually propagated to enhance the delivery performance, wherever a relay node receiving a message could forward it or carry it for long periods of your time, even once its arrival at the destination.

Such combination of message replication and long-run storage imposes a severe overhead on the mobile nodes that measure typically not only power energy-constrained but also buffer storage-limited. Thus, the message lifespan should be carefully turned thus to reduce the network resource consumption in terms of buffer occupation and power consumption whereas at the same time satisfy the desired delivery performance demand. It is noticed that there are some efforts in literature that specialize in the delivery likelihood study. Panagakis et al. in [9] analytically derived the message delivery likelihood of two-hop relay below a given time limit by approximating the cumulative distributed operate (CDF) of message delivery delay, with the belief that for any node pair the message may be with success transmitted whenever they meet one another. In [10], Whit beck et al. explored the impact of message size, message lifespan and link lifespan on the message delivery ratio (probability) of epidemic routing by treating the intermittently connected mobile networks (ICMNs) as edge-Markovian graphs, wherever every link (edge) is taken independent and simultaneously has the same transition probabilities between "up" and "down" status. Further it explored that impact of message scheduling and drop policies on the delivery likelihood performance of epidemic routing, and planned a distributed scheduling and drop policy supported applied math learning to approximate the optimum performance in [11]. The context of delay tolerant networks (DTNs) and the two-hop relay with $f$ cast in [12]-[22] has recently introduce the optimization issue of message delivery likelihood below specific energy constraints and message lifetime demand has been intensively self-addressed, within which the essential two-hop relay was adopted for packet routing and a wireless link becomes offered whenever two nodes meet one another.

\section{LITERATURE SURVEY}

A Mobile ad-hoc network (MANET) is a temporary network that dynamically establishes the network in the absence of fixed infrastructure which consists of self -organized mobile node which could freely join move around and leave the network. These mobile nodes are wireless connected through which they autonomously exchange control and manage information to for a network. MANET is flexible and selfautonomous wireless network. It is used to find many applications in daily information exchange, disaster relief, 
emergency response, daily information exchange, military troop communication, etc. In MANETS for long life and reliability the throughput capacity should be increased [1]. This work deals with each node as per capacity of a MANET, where the transmission power of every node is often controlled to adapt to such as transmission range and a generalized two-hop relay with restricted packet redundancy $\mathrm{f}$ is used for packet routing. Supported the construct of automatic feedback management and also the Markov Chain model, associate degree Inter-MANET Routing protocol known as InterMR that may handle the heterogeneousness and dynamics of MANETs is employed. Initial it defines associate degree Inter-MANET address theme supported a range of node attributes. Inter-MANET Routing protocol is also as called InterMR that used to handle the heterogeneity and dynamics of MANETs as per based on the concept of automatic feedback control and the Markov chain model. Based on a variety of node attributes initial it defines an InterMANET address scheme. Further it provides a seamless routing mechanism across heterogeneous MANETs without modifying the internal routing mechanisms in each MANET, by packet-level simulation, that the performance of InterMR will be improved by adaptive gateway assignment functionalities. Throughput parameter is established from the routing values. Increasing the transmission power of the nodes with this routing mechanism improves the capacity, and even at high packet rate increased throughput can be achieved.

For MANET, accurate \& robust capacity theory is the most difficult and important challenges in information theory which is explored by in [2]. The delay in the communication system is the major reason for the difficulties in the operational relevance of the capacity theory. In link, delay is related to the code word length. In the network, delay is measured by the traffic patterns, single-hop routing, channel access times, multi-hop routing, buffer occupation time, user mobility and retransmissions. For complex message delivery method in MANET is possible by making use of Markov process model to unfold the messages. The message lifespan is strictly tuned and decreased to realize the most delivery quantitative relation. Two hop relay with FEC coding rule is employed. The results clearly show that by reducing the delay and thereby up the delivery quantitative relation, improvises the turnout capability and network performance.

In [3] Groenevelt et al consider a stochastic model. It is accurately models the message delay in mobile ad hoc networks where nodes relay messages. This model introduced two input parameters: the number of nodes and the parameter of an exponential distribution which describes the time until two random mobiles come within communication range of each other. It characterizes the delay incurred by a message in MANET. Two protocols such as the two-hop and the unrestricted multi copy protocols obtained for allocation of the number of replicas of the message at the time the message is delivered to the destination node. It is shown that the model accurately predicts the message delay for both relay strategies for a number of mobility models. Based on the theoretical framework, we derive both a closed-form expression and an asymptotic approximation for the corresponding message delivery probability.

Communication networks are traditionally assumed to be connected. However advanced wireless applications such as vehicular networks, pocket-switched networks, etc., coupled with volatile links, node mobility, and power outages, will require the network to operate despite frequent disconnections. At the end, routing techniques have been recommended, where a node may store-and-carry a message for some time, until a new forwarding opportunity arises. Though variety of such algorithms exists in [4], most focus on homogenous setting nodes. However, in several unreal applications, participating nodes might include handhelds, vehicles, sensors, etc. In this paper, we address the problem of routing in intermittently connected wireless networks comprising multiple classes of nodes. We show that suggest solutions, which perform well in homogeneous scenarios, are not as competent in this setting. To this end, we propose a class of routing schemes that can identify the nodes of "highest utility" for routing, improving the delay and delivery ratio by four to five times. Additionally, based on fluid models we propose to analyze the performance of various opportunistic routing strategies, in heterogeneous settings.

According to the topology changes in mobile ad hoc networks, it has been a big challenge to develop a routing protocol and optimize routing paths. More generally, the geographic routing is to be more efficient and robust in a dynamic environment [23]. However, the use of proactive fixed-interval beaconing to distribute local positions introduces high overhead when there is no traffic and cannot capture the topology changes under high mobility. It is also difficult to preset protocol parameters correctly to fit in different environments. In the sense, develop two selfadaptive on-demand geographic routing schemes which build efficient paths based on the need of user applications and adapt to various scenarios to provide efficient and reliable routing. On-demand routing mechanism in both protocols utilizes to reduce control overhead compared to the proactive schemes which are normally adopted in current geographic routing protocols. Additionally, our route optimization scheme adapts the routing path according to both topology changes and actual data traffic requirements.

This paper present a geographic routing which is one type of routing ad hoc and sensor networks with a specific routing algorithm based on a synthesis of the greedy forwarding and face routing approaches. It provides an algorithmic analysis of the presented algorithm from both a worst-case and an average-case perspective [24]. The GOAFR geographic routing algorithm is a combination of the greedy forwarding and face routing approaches. Using face routing algorithm is proved to require at most steps where is the cost of a shortest path connecting the source and the destination. Employing greedy forwarding, the algorithm also becomes efficient in average-case networks. In most GOAFR algorithm can be considered a synthesis of simplicity and average-case efficiency on the one hand and correctness as well as asymptotic worst-case optimality on the other hand.

It has been a challenge itself in the design of wireless ad-hoc networks is the restricted availability of the energy resources. Basing their resources limited in wireless networks than in wired networks. However, the geographic routing protocols are position based protocols to be are more scalable and reliable routing protocols with their forwarding decisions based on the local topology. Even though geographic routing has many advantages and has shown a vast prospective, the inaccurate knowledge of local geographic topology can lead to greatly affect routing performance. Even through, this not only to high packet delivery latency, more collisions and energy consumption but can also result in a routing failure. They act as proficient forwarding choices, based on the positions of their immediate neighbors [25]. To maintain neighbor position, periodic broadcasting of beacon packets that contain the geographic position of the nodes is a well- 
liked method used. In this paper, low Energy Self Adaptive (LESA) on-demand geo-routing reduces control overhead compute up to the proactive schemes which provides reliable routing at the same time, make the lesser amount of energy utilization.

\section{PROPOSED APPROACH FRAMEWORK AND DESIGN}

\subsection{Architecture}

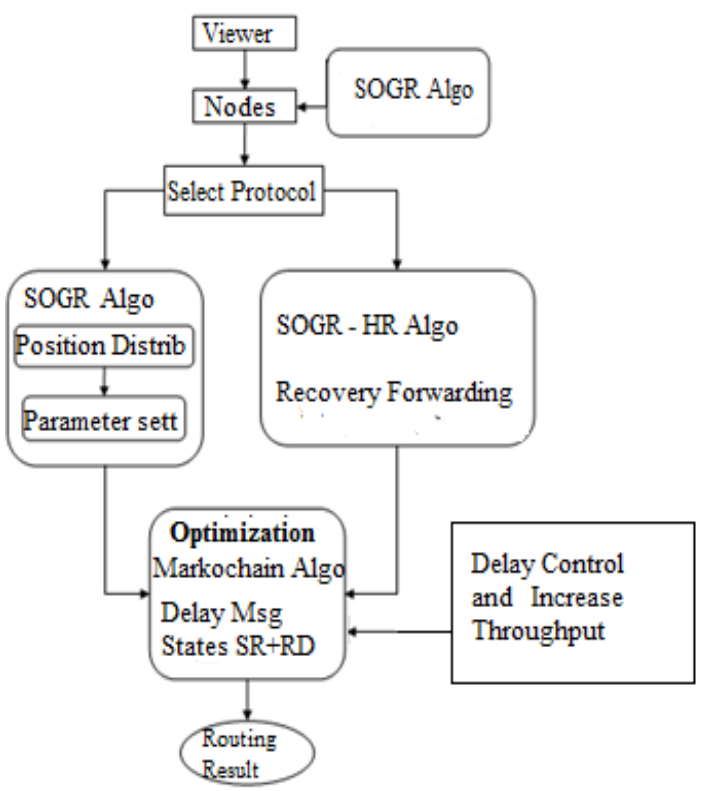

Fig 1: Proposed System Architecture

\subsection{Process Flow}

The architecture of a system is shown in figure. The components of system are:

Viewer: It is the front end of the system. User has view the output results and configure the system using the viewer. User has authority to find the location of present node as well as node movement. It also configures the location service with the termination timer for the cached entries.

The system consists of three routing methodologies such as SOGR-HR, SOGR-GR, AND SOGR-LESA.

\subsubsection{SOGR-HR}

Greedy Forwarding: Greedy Forwarding approaches is that, at the each stage visit an unvisited node nearest to the current node [10]. Thus, every node forwards the message to the neighbor that's best suited from a local point of view. Greedy strategy is chooses neighbor that have minimizes the distance to the destination in each step (Greedy).

Parameter Settings: Each node can determine and adjust the protocol parameter values independently according to different network environments, information traffic conditions and mobile nodes' to their own needs using each protocol with parameter settings.

\subsubsection{SOGR GR}

(Geographic primarily based reactive mechanism) this protocol is avoid the collision, it primarily depend upon just one hop neighbour's positions. The SOGR GR and hour the two protocols are primarily have the necessary parameter. Set there parameter get best performance. The on top of two protocols are want to forward the packet from supply to destination while not collision however it will give most finish to finish delay.

\subsubsection{SOGR-LESA}

SOGR-LESA algorithm apply on nodes after it deploy. It requires modification to route look up process at each node at any time, only those entries in a nodes routing that correspond valid next-hops.

Optimization: Optimization has improves transmission path. Their schemes are applied in order that a forwarding node and its neighbors will collaborate to adapt the path to each topology change and traffic demand.

\subsubsection{Node Creation}

Number of mobile nodes created and placed within the network. Nodes will be added and deleted from the network at any time dynamically. Connection to a node is created only if another node requests the actual node. Frames are exchanged between the nodes as they are available at intervals reach vary of 1 another. The MANET considered as a Torus that is split into $\mathrm{m}^{*} \mathrm{~m}$ equal cells with $\mathrm{n}$ mobile nodes. Time is slotted and node randomly roams from cell to cell. Node movements are independent from one time slot to a different slot in order that they measure reshuffled at every time slot. Protocol interference model is employed to support synchronal transmission.

\subsubsection{Marko chain Model}

A transient state $(i, j)$ is denoted where $S$ delivers $i$ frames and $D$ receives $\mathrm{j}$ frames. There are four possible transient States.

Case 1: - source-to-relay $\|(\mathrm{SR})$ transmission only.

Case 2: - relay-to-destination $\|(\mathrm{RD})$ transmission only.

Case 3: both - source-to-relay \| and -relay-to destination\| (SR+RD) transmissions.

Case 4: - source-to-destination $\|(\mathrm{SD})$ transmission only.

\subsection{Mathematical Model}

\subsubsection{Input}

Random nodes for Communication and Packet Delivery

\subsubsection{Message Lifetime probability}

For the tagged flow, message generated at the source node $\mathrm{S}$ is assumed to have erasure coded into total $w . \beta$ frames, the delivery delay of the message is defined as the time elapsed between the time slot when $\mathrm{S}$ starts to deliver the first frame of the message and the time slot when the destination node receives $w$ distinct frames of the message.

$\emptyset(w, \beta, \tau)=\operatorname{Pr}(T d>\tau)=\sum_{t=1}^{r} \operatorname{Pr}(T d=\tau)$

Where, $\tau$ is lifetime constraint

$\mathrm{W}=$ Erased Coded, $\mathrm{d}=$ transition state

\subsubsection{Transition Probability}

$\mathrm{B}=(\mathrm{bi}) \mathrm{d}^{*} 1$

\subsubsection{Message Delivery Probability}

$\emptyset(\omega, \beta, \tau)=\sum_{t=1}^{\tau}$ e. $Q^{t-1} . B$

\section{RESULTS}

Following figures are showing results for practical work which is done. Following figure indicates that the energy 
efficient on-demand Geographic routing for mobile ad-hoc networks. They provide reliable and energy efficient feature to increase the message lifetime of the network.

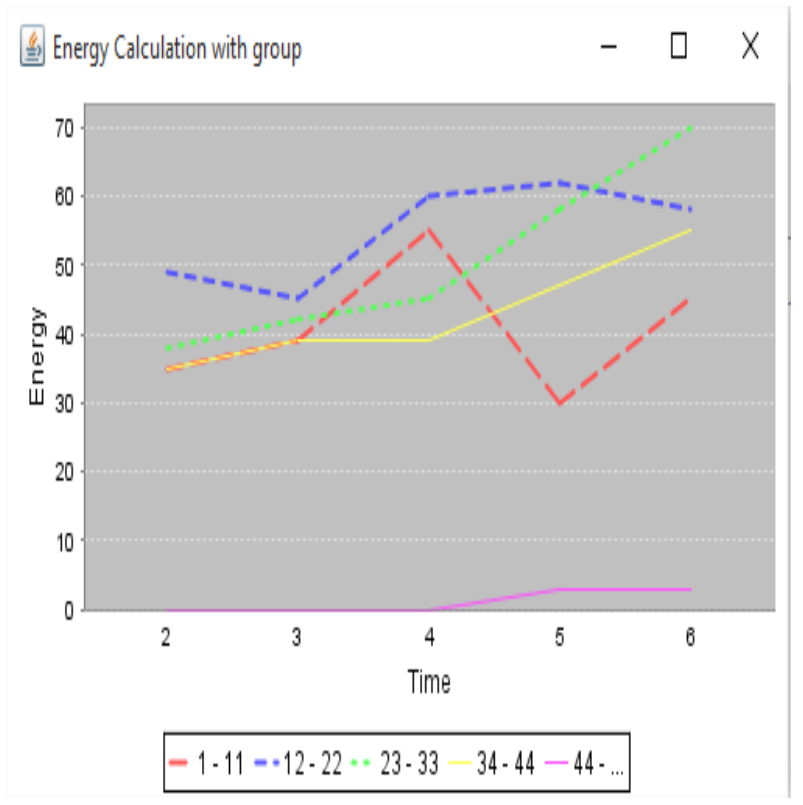

Fig 2: Energy Calculation Graph

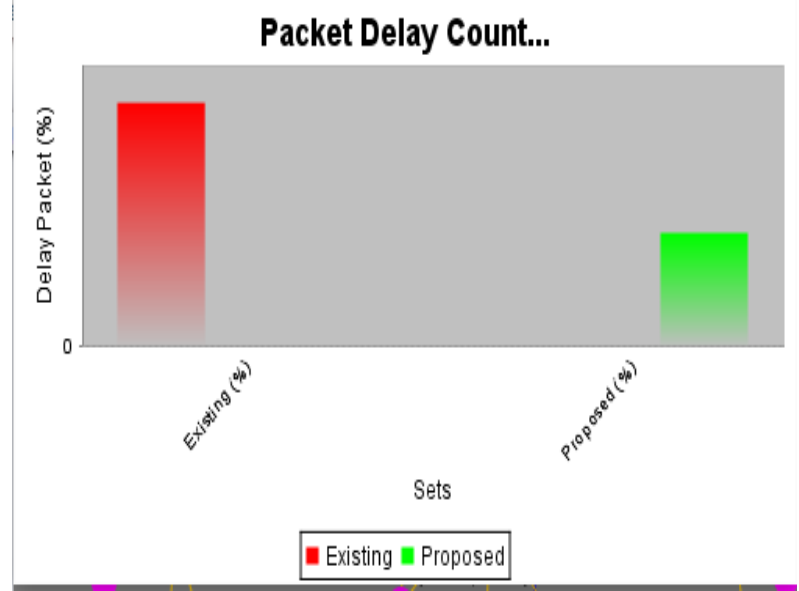

Fig 3: Packet Delay Graph

Table1. Energy Calculation Values Table

\begin{tabular}{|c|c|c|c|c|c|}
\hline \multirow{2}{*}{ Energy } & \multicolumn{5}{|c|}{ Time } \\
\cline { 2 - 6 } & $\mathbf{2}$ & $\mathbf{3}$ & $\mathbf{4}$ & $\mathbf{5}$ & $\mathbf{6}$ \\
\hline Cluster 1 & 35 & 39 & 54 & 30 & 46 \\
\hline Cluster 2 & 49 & 46 & 60 & 62 & 59 \\
\hline Cluster 3 & 38 & 43 & 46 & 58 & 71 \\
\hline Cluster 4 & 35 & 39 & 39 & 47 & 56 \\
\hline Cluster 5 & 0 & 0 & 0 & 3 & 3 \\
\hline
\end{tabular}

Table2. Packet Delay Table

\begin{tabular}{|c|c|c|}
\hline & Existing System & Proposed system \\
\hline Packet Delay & 0.892 & 0.451 \\
\hline
\end{tabular}

\section{CONCLUSION}

This paper works on the delivery likelihood performance in a two-hop relay mobile ad hoc network. A general Marko chain theoretical framework was derived to characterize the message delivery process, which might even be used to analyze the delivery likelihood performances under different routing protocols. The new theoretical framework approach consider, closed-form expressions were more derived for the delivery likelihood under a given message life time and message size. The framework will be used with efficiency model the message delivery method and therefore accurately characterize the delivery likelihood performance there. Another interesting finding of work is that the energy efficient on-demand Geographic routing for mobile ad-hoc networks. There has been to provide reliable and energy efficient feature to increase the lifetime of the network. The results indicate that for a two-hop relay MANET with erasure coding, having a limiting performance for the delivery likelihood which is determined by the management parameters of message size $\omega$ and message period of time $\tau$. Another interesting finding of work is that the thought of MANETs truly exhibit very similar behaviors in terms of delivery likelihood below totally different node mobility models, just like the i.i.d., random waypoint. The main idea is to add to provide in terms of determine a suitable message lifetime, so as minimize per node buffer occupation and power consumption while simultaneously meet the delivery performance requirement.

\section{REFERENCES}

[1] A. Goldsmith, M. Effros, R. Koetter, M. Medard, A. Ozdaglar, andL. Zheng, "Beyond Shannon: the quest for fundamental performancelimits of wireless ad hoc networks," IEEE Commun. Mag., vol. 49,no. 5, pp. 195205, May 2011

[2] J. Andrews, S. Shakkottai, R. Heath, N. Jindal, M. Haenggi, R. Berry,D. Guo, M. Neely, S. Weber, S. Jafar, and A. Yener, "Rethinkinginformation theory for mobile ad hoc networks," IEEE Commun. Mag., vol. 46, no. 12 , pp. 94-101, Dec. 2008.

[3] R. Groenevelt, P. Nain, and G. Koole, "The message delay in mobile adhoc networks," Performance Evaluation, vol. 62, no. 1-4, pp. 210-228,Oct. 2005.

[4] X. Zhang, G. Neglia, J. Kurose, and D. Towsley, Performance modelling of epidemic routing, in Proc. 2006 IFIP Network.Conf .

[5] A. A. Hanbali, P. Nain, and E. Altman, "Performance of ad hoc networkswith two-hop relay routing and limited packet lifetime," in Proc. 2006Value tools Conf.

[6] A. A. Hanbali, P. Nain, and E. Altman , "Performance of ad hoc networks with two-hop relay routingand limited packet lifetime-extended version," Performance Evaluation,vol. 65, no. 6-7, pp. 463-483, June 2008.

[7] J. Liu, X. Jiang, H. Nishiyama, and N. Kato, "Performance modelling for two-hop relay with erasure coding in Manet," in Proc. 2011 IEEEGLOBECOM.

[8] J. Liu, X. Jiang, H. Nishiyama, and N. Kato, "Groupbased two-hop relay with redundancy in magnets," in Proc.2011 IEEE HPSR Conf.

[9] A. Panagakis, A. Vaios, and I. Stavrakakis, "Study of two-hop messagespreading in DTNS," in Proc. 2007 WiOpt. 
[10] J. Whitbeck, V. Conan, and M. D. de Amorim, "Performance of opportunistic epidemic routing on edgeMarkovian dynamic graphs,"IEEE Trans. Commun., vol. 59, no. 5, pp. 1259-1263, May 2011.

[11] A. Krifa, C. Barakat, and T. Spyropoulos, "Message drop and schedulingin DTNS: Theory and practice," IEEE Trans. Mobile Compute., to bepublished

[12] E. Altman, T. Basar,and F. D. Pellegrini, "Optimal monotone forwarding policies in delay tolerant mobile ad-hoc networks," in Proc. 2008 Inter-Perf Conf.

[13] E. Altman T. Basar and F. D.Pellegrini, "Optimal control in two-hop relay routing," IEEE Trans. Autom.Contr., vol. 56, no. 3, pp. 670-675, Mar. 2011.

[14] E. Altman and F. D.Pellegrini, "Forward correction and fountain codes in delay tolerant networks," in Proc. 2009 IEEE INFOCOM.

[15] E. Altman, F. D. Pellegrini, and L. Sassatelli, "Dynamic control ofcoding in delay tolerant networks," in Proc. 2010 IEEE INFOCOM.

[16] E. Altman, G. Neglia, F. D. Pellegrini, and D. Miorandi, "Decentralizedstochastic control of delay tolerant networks," in Proc. 2009 IEEEINFOCOM.

[17] E. Altman, A. P. Azad, T. Basar, and F. D. Pellegrini,"Optimal activation and transmission control in delay tolerant networks," in Proc. 2010 IEEE INFOCOM.
[18] F. D. P. WissamChahin and, R. EI-Azouzi, and A. P.Azad, "Blindonline optimal forwarding in heterogeneous dtns," in Proc. 2011 IFIPWireless Days.

[19] C. Singh, A. Kumar, and R. Sundaresan, "Delay and energy optimaltwo-hop relaying in delay tolerant networks," in Proc. 2010 WiOpt.

[20] R. EI-Azouzi, F. D. Pellegrini, and V. Kamble, "Evolutionary forwardinggames in delay tolerant networks," in Proc. 2010 WiOpt.

[21] R. EI-Azouzi, F. D. Pellegrini, and V. Kamble, "Evolutionary forwardinggames in delay tolerant networks," in Proc. 2010 WiOpt.

[22] A. Vahdat and D. Becker, "Epidemic routing for partially connected adhoc networks," Tech. Rep. CS200006, Duke Univ., Durham, NC, Apr.2000.

[23] X. Xiang, Z. Zhou, and X. Wang, "Self-Adaptive On Demand Geographic Routing Protocols for Mobile Ad Hoc Networks," IEEE TRANSACTIONS ON MOBILE COMPUTING, vol. 11, no. 9, September 2012

[24] F. Kuhn, R. Wattenhofer, and A. Zollinger, "An Algorithmic Approach to Geographic Routing in Ad Hoc and Sensor Networks," IEEE/ACM Trans. Networking, vol. 16, no. 1, pp. 51-62, Feb. 2008.

[25] Y. Li, Y. Yang, and X. Lu, "Routing Metric Designs for Greedy, Face and Combined-Greedy-Face Routing" Proc. IEEE INFOCOM 09, Page(s):64 -72, Apr. 2009. 